\title{
EDDY CURRENT EFFECTS IN AN OPPOSITE-FIELD SEPTUM
}

\author{
K. Fan ${ }^{\#}$ I. Sakai, Y. Arakaki, KEK, Tsukuba, Ibaraki, Japan
}

\section{Abstract}

A large aperture, thin-septum, high-field opposite-field septum magnet has been developed for the injection of $50 \mathrm{GeV}$ main ring of J-PARC. Due to the eddy current generated in septum conductor, magnet yoke and magnet end plate, the field quality was degraded. In the paper, eddy current effects on the field distribution are discussed. Correction methods and experiment results are introduced.

\section{INTRODUCTION}

The J-PARC is a high beam intensity accelerator project. A large aperture septum magnet is required to accommodate the large size low energy injected beam. To keep a sufficient beam clearance avoiding serious residual radiation problems, the septum is required less than 8 $\mathrm{mm}$. To cope with these requirements, a large-aperture, thin-septum, opposite-field septum magnet has been developed [1,2]. Fig.1 shows the scheme. For injection beam, the gap field is doubled by the two sub-bending magnets. The field for circulating beam will be cancelled completely. The basic parameters are listed in table 1 .

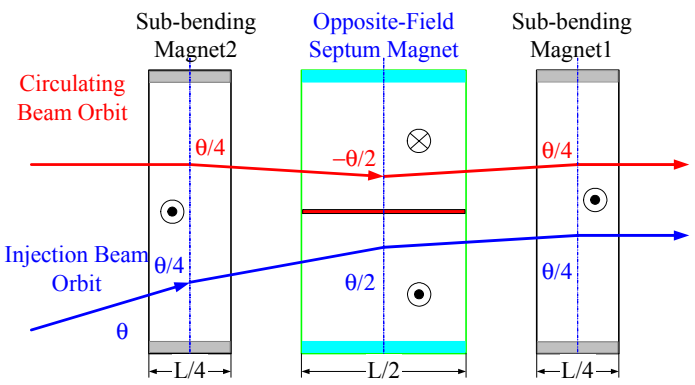

Figure 1: Principle of opposite-field septum magnet.

Table 1: Basic Parameters of Opposite-field Septum

\begin{tabular}{|l|l|}
\hline Parameters & Value \\
\hline Gap field & 0.6 Telsa \\
\hline Aperture & $150 * 120 \mathrm{~mm}$, opposite-field septum \\
& $400 * 120 \mathrm{~mm}$, sub-bending magnet \\
\hline Length & $\begin{array}{l}700 \mathrm{~mm}, \text { opposite-field septum } \\
350 \mathrm{~mm}, \text { sub-bending magnet }\end{array}$ \\
\hline Septum & $8 \mathrm{~mm}$ \\
\hline
\end{tabular}

\section{EDDY CURRENT ON SEPTUM}

Fig.2 shows the cross section of the opposite-field septum magnet. The septum conductor, which has been optimized to a special geometry, is installed in the middle of magnet aperture. When excite by a current pulse, high transient magnet field imposes on the septum conductor and generates eddy current, which is shown in Fig.2.

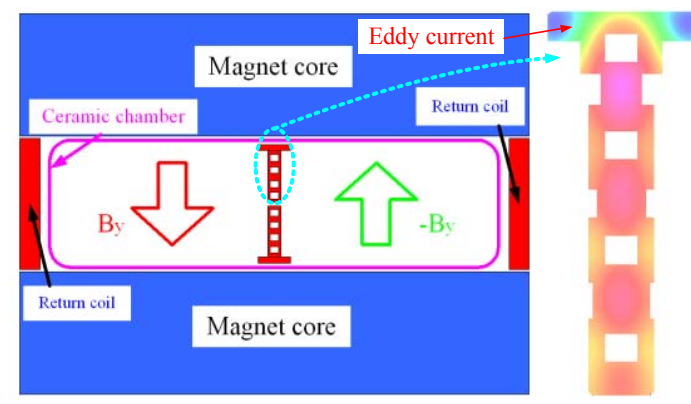

Figure 2: Opposite-field septum.

The eddy current redistributes the current on the septum and thus changes the gap field distribution near the septum as shown in Fig.3. Measurement results agree with the calculation well.

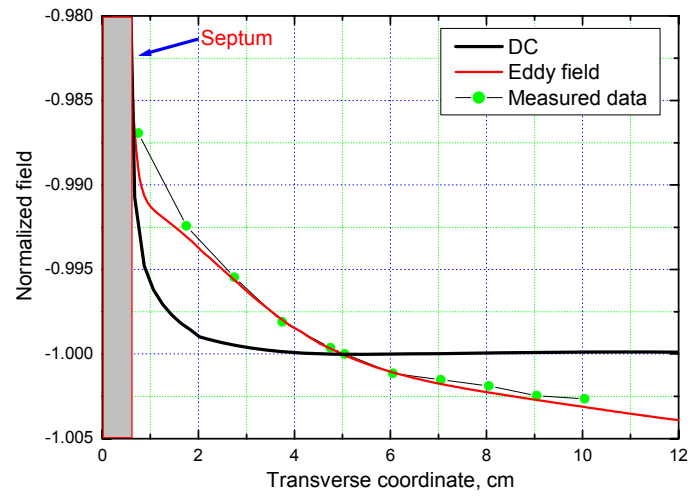

Figure 3: Gap field degradation due to eddy current.

\section{EDDY CURRENT ON MAGNET YOKE AND END PLATE}

In a laminated iron magnet, considerable eddy current can only be generated at the magnet ends, especially for a large aperture magnet. These eddy currents not only consume energy causing thermal problems but also generate "eddy field".

\section{Eddy Current on Opposite-field Septum Magnet}

With OPERA 3D codes, the eddy current distribution in the opposite-field septum magnet can be simulated as shown in Fig.4. The eddy currents are generated at magnet ends mainly due to the considerable field components normal to the magnet ends. Narrow slits are fabricated at the magnet ends to decrease the eddy current. 


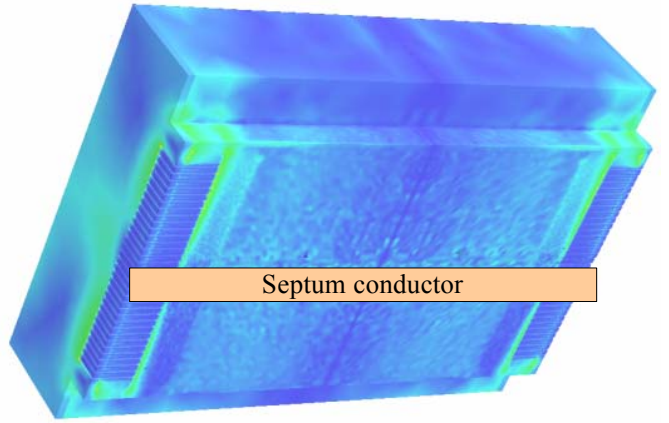

Figure.4: Eddy current distribution on opposite-field septum.

Due to the eddy current effects, the degradation of gap field is unavoidable. Moreover, the gap field is also time delayed, which indicates that the circuit for eddy current contains reactance. Fig.5 shows the gap field degradation.

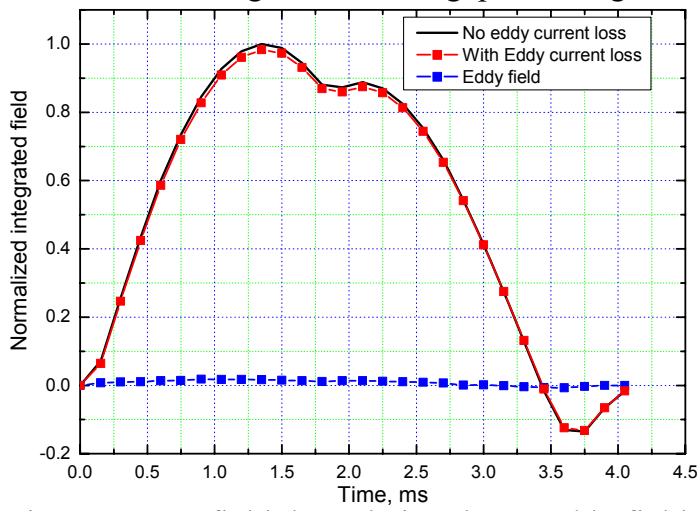

Figure 5: Gap field degradation due to eddy field.

\section{Eddy Current on Sub-bending Magnet}

Fig. 6 shows the eddy current distribution on the subbending magnets. The maximum eddy current is about 2 times higher that that of the opposite-field septum. This is because the opposite-field septum magnet yoke is divided into two regions. As a result, the effective lamination dimension for the circulating eddy current decreases to half and, the resistance is nearly doubled, which decreases the eddy current losses accordingly. Fig. 7 shows the gap field degradation due to the eddy field.

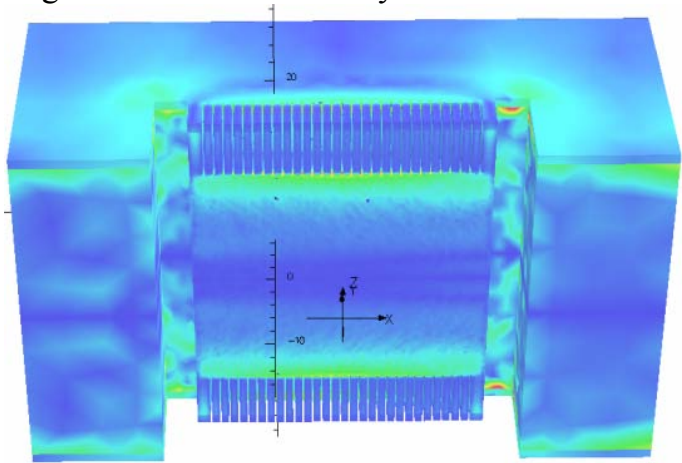

Figure 6: Eddy current distribution on sub-bending magnet.

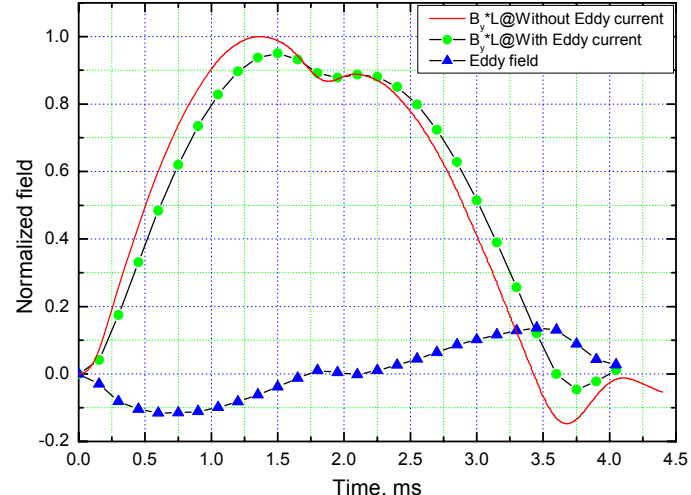

Figure 7: Gap field degradation due to eddy field.

\section{TRANSVERSE FIELD COMPENSATION}

The transverse field contains $Q$ field due to the eddy current effects. Beam optics study shows that the Q field can cause $30 \% \quad \beta$ function modulation. Thus, field compensation is needed.

\section{Local Compensation Method}

In order to compensate the field distribution, one direct way is to reduce the eddy current on the spot, which can be realized by increasing the magnet gap. Fig. 8 compares the transverse field distribution with different gap height. It shows that when the gap increase about $3.8 \mathrm{~mm}$, the transverse field distribution close to uniform. However, increasing the magnet gap also decrease the gap field, which increase the burden of power supply.

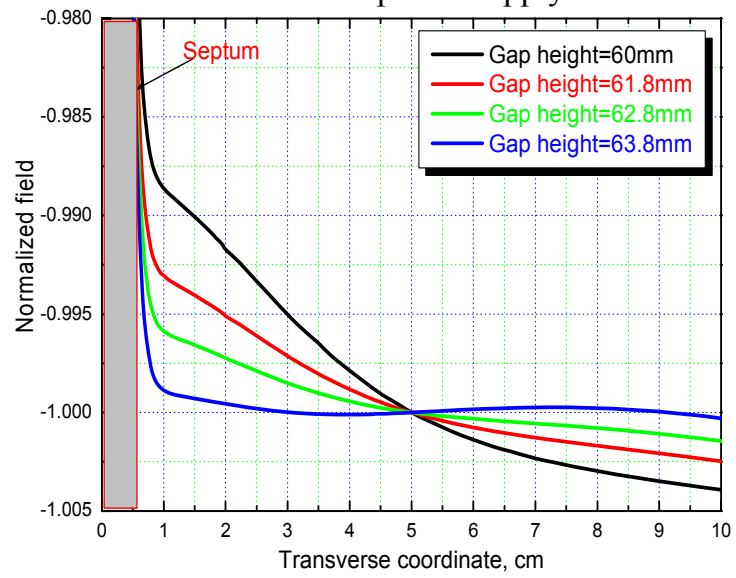

Figure 8: Transverse field compensation.

\section{External Compensation Method}

In this way, the opposite-field septum remain untouched and the "Q" field still exist locally. However, a compensation " $-Q$ " field is provided by the two subbending magnets. By proper tilting the two sub-bending magnets, the $\mathrm{Q}$ field can be diminished and the integrated transverse field quality can be improved a lot.

However, this method is not allowed if the "Q" field is considerable large. Otherwise, beam optics might be affected. 


\section{Combined Compensation Method}

In practice, two methods are combined to compensate the transverse field quality. Fig.9 shows the calculation and measured results.

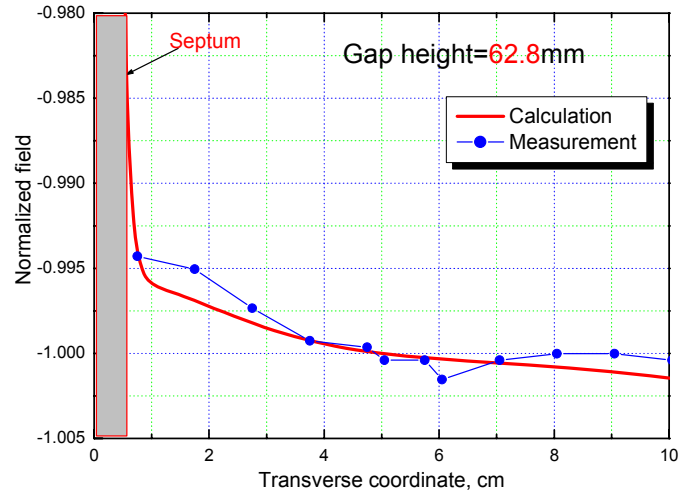

Figure 9: Transverse field distribution after compensation.

\section{LEAKAGE FIELD COMPENSATION}

In ideal case, the integrated field for the circulating beam is zero. However, due to the difference of eddy current losses, the gap field balance between the three sub-magnets is broken. Therefore, the field for circulating beam cannot be cancelled completely and residual gap field existed. With a long search coil, the residual field was measured as shown in Fig.10. 3D calculations agree with the measurement results well.

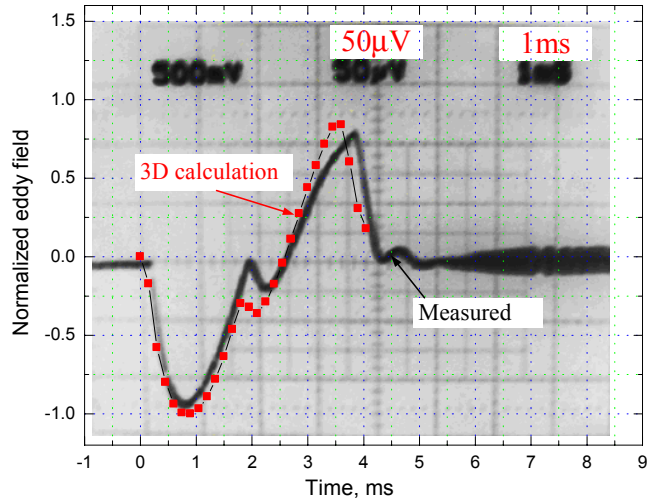

Figure 10: Leakage field comparison.

The effects of eddy current losses can be viewed as a chain of $\mathrm{R} / \mathrm{L}$ circuit parallel connects to the main coil. Simply, the chain is truncated to two orders circuit as shown in Fig.11.

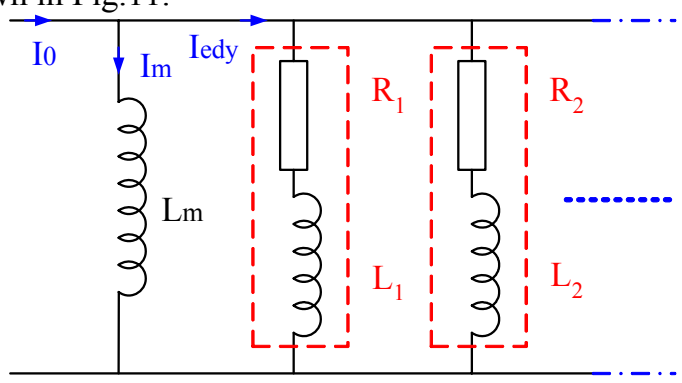

Main coil Magnet end plate/lamination

Figure 11: circuit model of eddy current loss
Since the two sub-bending magnets have more magnet ends and have large dimension, the corresponding parameter of $\mathrm{R} / \mathrm{L}$ is smaller, consequently the eddy current loss is larger. Thus, to compensate these excessive eddy current losses, additional "eddy current losses" have to be introduced to the opposite-field septum, which can be realized by using a pair of self-induced back-leg windings. Fig.12 illustrates the scheme.

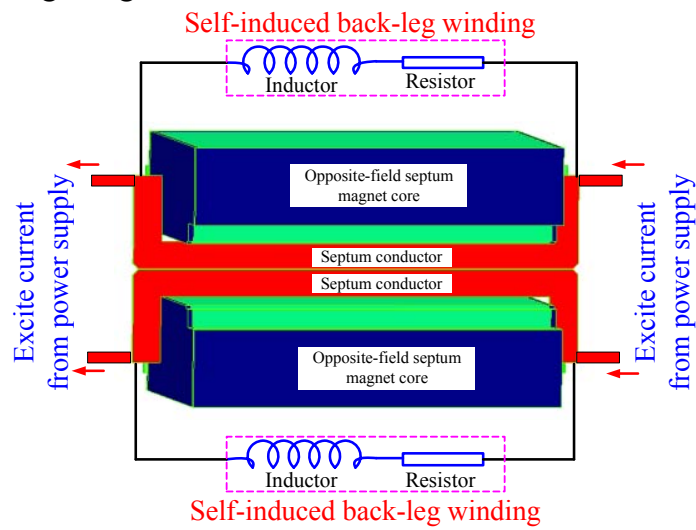

Figure 12: Self-induced compensation.

The parameters of the self-induced back-leg windings can be determined roughly by $3 \mathrm{D}$ simulation. In practice, together with "trial and errors", the leakage field can be suppressed greatly. Fig. 13 shows the measured result.

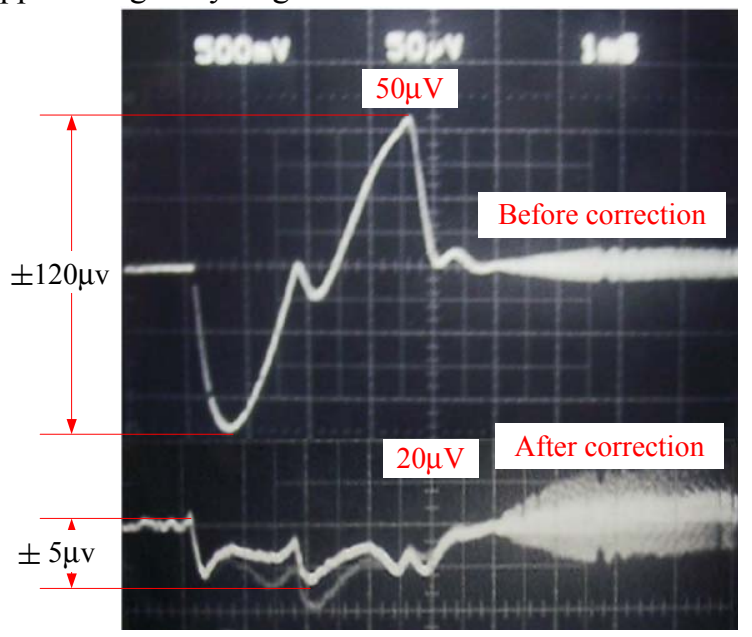

Figure 13: Leakage field suppression.

\section{SUMMARY}

The opposite-field septum provides a new solution to design a large-aperture, thin-septum, high-field septum magnet. Since it is the first of this kind septum magnet, further calculations and experiments are needed to improve the magnet performance.

\section{REFERENCES}

[1] I. Sakai et al, Opposite field septum magnet system for the J-PARC $50 \mathrm{GeV}$ ring injection, EPAC04

[2] I. Sakai et al, OppositeField Septum Magnet System for the J-PARC. Main Ring Injection, MT19 